\title{
Research on Quality Control Technology of Clinical Laboratory
}

\author{
Zhimei Zhang \\ Luohe Medical College, Luohe, Henan, 462000, China
}

\begin{abstract}
With the development of modern medicine, medical testing technology is also developing rapidly and many diseases, diagnosis, treatment and prognosis rely on the results of medical tests. Medical examination plays an important role in clinical medicine work, and the results of medical tests directly affect the diagnosis of patients with diseases. Quality control is the key to improve the quality of clinical testing. The purpose of this paper is to analyze the quality control measures of clinical medicine, to improve the accuracy of test results, to ensure the correct rate of disease diagnosis and better serve the patients.
\end{abstract}

Keywords: quality control technology, clinical laboratory

\section{Introduction}

With the development of social progress, the rapid advancement of medical testing technology, broaden the vision of clinical medicine, expanding awareness of the depth and breadth of disease, clinical diagnosis and differential diagnosis of disease diagnosis, efficacy monitoring and prognosis, epidemiological investigation and the environment Monitoring, health counselling and disease prevention, clinical research and other aspects play an indispensable role. Whether the result of clinical examination is accurate or not will directly influence the judgment of the disease and the correctness of the diagnosis and treatment plan. Quality control is an effective way to improve the quality of clinical laboratory testing, quality assurance system has gradually matured into an important part of laboratory construction and management. The quality of clinical examination directly affects the diagnosis of patients, affecting the quality of 
medical care and medical security. Therefore, we must strengthen the quality of clinical medical testing measures to ensure the specimen before analysis, analysis, analysis, after the whole quality control. In order to protect patients' rights and medical quality and prevent misdiagnosis, we must improve the quality of clinical tests, carry out comprehensive quality control on samples, ensure the completeness of physical and pathological samples and prevent the quality of specimen collection and analysis Differences, affecting the results, but not the right response to the patient's condition. In this paper, analysis of clinical testing results of the factors that affect the authenticity of focus, analysis of quality control of clinical laboratory tests.

\section{The concept of quality control of clinical testing}

The requirements of the clinical testing centre is to provide the basis for testing quality, quality to meet the application requirements, we must implement quality assurance, quality assurance system includes two aspects, namely the determination of analysis centre and before and after the quality control, attention to laboratory management Quality control of the first steps, only a good laboratory management, in order to ensure accurate test results, quality control steps in three ways, first, the inspection section of the inspection staff must establish a good relationship with the clinician, inspection personnel to For the doctor to explain the correct impact test results of various factors to ensure that the test results can guide the doctor to make the right decisions, and second, the collection of specimens to be careful to ensure the correctness of the specimen $\%$ eligibility and timely delivery of laboratory, Third, the strict implementation of the calculation of the registration and the development of reports and other measurement centre quality control, to avoid experimental error occurs, any of these three steps of a problem, will lead to test results error or failure, so the clinical quality control of each test Steps must be strictly enforced.

\section{The problems and shortcomings of clinical testing quality control}

\subsection{The problem caused by specimens}

Specimen collection substandard requirement is an important reason for the error of test results. Note that the position of blood on the test results, the use of tourniquet time shall not exceed 1min; grasp the correct method and order of vacuum blood collection in order not to avoid the interference of factors, attention to sampling the best time; Collection of samples to be done after the check system strictly; urine specimens taken, the container should be clean, specimens should be fresh, not mixed with secretions, semen and so on. 
Patient factors are mainly divided into fixed factors and variable factors. Fixed factors are mainly the patient's age, gender, ethnic and geographical and so on. Variables include diet, exercise, and the effects of drugs on test results. Patients must be asked to collect samples in quiet or normal activity, preferably before taking some drugs. Collection of specimens of improper operation, such as directly from the infusion arm blood, can cause blood samples were diluted; after taking the blood is not unplug the needle into the blood test tube can cause hemolysis of blood samples.

Test factors can be divided into non-qualified specimens and random use of anticoagulants. At present, the blood test has been fully automated blood cell analyzer and automatic biochemical analyzer. Take $5 \mathrm{ml}$ of blood can do liver function, renal function, protein, blood sugar more than 30 biochemical series, so the specimen will directly affect the test results of the accuracy of the failure.

Specimens stored in vitro after the special requirements, to ensure that there are hand delivery, delivery process to be safe, to prevent shock, to prevent samples from contamination, to ensure that within the specified time to the testing laboratory. Specimens suspected of high risk should be transported in strict accordance with the relevant regulations and be strictly packed to prevent transmission to others. Upon receipt of specimens by the laboratory should be immediately checked by the hand. On non-conforming specimens, should be timely and doctors to contact, explain the reasons, and make records.

Serious hemolysis, chylous blood should be noted in the report of the results; urine specimens are not fresh or add the blood to the blood sample, the blood sample should be re-take blood, The samples of the preservative do urine analysis, can lead to the results should not have abnormalities, should ask the patient whether to take vitamin $\mathrm{C}$ and other test results of the drug.

\subsection{The problem caused by equipment and reagents}

Equipment and reagents caused by the problem mainly in: First, the test personnel on the use of the instrument is not very understanding of the function, and the reagent is not in accordance with the instructions to change the different parameters, resulting in experimental results systematic error. Second, it is the experimental results error caused by poor maintenance of the instrument. Third, the use of reagents and equipment requirements do not affect the experimental results. The solution is to strengthen the custody, troubleshooting, a variety of instruments and reagents are equipped with a proper maintenance and custody, the operator proficiency in instrument performance, measurement methods and simple troubleshooting methods.

\section{The problem caused by human factors}

Medical testing work after all or people's work, even if there are advanced equipment and equipment are still mixed with human factors. Therefore, the inspectors, the work to maintain adequate patience and careful, due to psychological factors that cause errors in the inspection staff should carefully analyze the reasons for reasonable recommendations, pointed out that should be 
improved and the direction of future efforts. In order to improve the credibility of the test results, to better reflect the actual situation of the object was seized, requiring inspectors to strengthen their self-cultivation, improve professional quality, cultivate a good medical ethics, in a rigorous and scientific attitude to do their own Work together to strengthen the clinical exchanges and cooperation with clinicians to improve the accuracy and credibility of the test results and work together.

\section{The measures to improve the quality control techniques of clinical testing}

\subsection{Health administrative departments should promptly improve the clinical test operation standards}

All clinical test items should have standard operating procedures, instrument maintenance, calibration and use should also develop standard operating procedures. But the rapid advances in science and technology has brought a lot of emerging clinical testing equipment, the emergence of clinical testing projects is also increasing. China's health administrative departments should follow the latest inspection techniques, equipment and projects, develop appropriate operational procedures. Improve the quality of clinical testing is a difficult, complex systems engineering. In the work, the need to have rules to follow, there are chapters to keep. Therefore, to improve the quality management of the various rules and regulations, in practice, actively listen to the inspectors, clinicians and hospital management staff comments and suggestions, and constantly improve the development of more targeted clinical laboratory management standards and norms, improve the compliance of the test personnel in practice, the interpretability of clinical test results, and the efficiency of hospital management. At the same time, we should give full play to the role of the administrative departments and management departments to ensure that the quality control technology is fully implemented, in strict accordance with the quality control requirements of the experiment and assessment, strengthen the concept of quality control and professional ethics education, thus promoting the improvement of inspection quality.

\subsection{Medical institutions should strengthen the clinical testing management}

Some hospitals in the clinical departments, pharmacy, isotope and other departments to establish the same or similar clinical testing projects, staffing, environmental conditions, quality assurance measures and the inspection department there is a gap, which not only a waste of resources, Unification and interpretation of test results. Therefore, the hospital should focus on clinical testing items set, unified management and resource sharing, to ensure the quality of clinical testing. How to reduce the cost of laboratory operation and provide clinicians with the most abundant, accurate and effective information service 
under the premise of ensuring the quality of laboratory services and improving the efficiency of monitoring, has become the core problem of clinical test cost management. Clinical examination of the economic benefits is based on cost accounting, in order to strengthen the cost accounting management, inspection costs must be analyzed, while reasonable calculation. The cost of clinical testing, including material costs and labour costs, can be divided into direct costs and indirect costs, the actual cost of a test project should include the actual direct materials, direct labour costs and indirect costs. Indirect costs for clinical testing include rents for clinical laboratories, insurance, property taxes, depreciation of equipment, maintenance and repair of equipment, utilities, heating and water. In the market economy under the influence of this lever, the high cost of laboratory testing of poor quality in the competition will be eliminated.

Indoor quality control can detect and control the precision and accuracy of daily work, and improve the uniformity of the results of the routine assay. Laboratory monthly indoor quality control data should be statistical processing, preservation, but also on the month of indoor quality control data, the mean, standard deviation, coefficient of variation to evaluate the previous month with the average, standard deviation Whether there is a significant difference. If there are significant differences, it is necessary to control the mean value, standard deviation to be modified, and to re-design quality control methods. Interlaboratory quality assessment is the use of inter-laboratory comparison to determine the clinical testing capacity and its purpose is to ensure a high level of clinical testing. All clinical testing programs should participate in the interlaboratory quality assessment activities organized by the Clinical Laboratory Centre and develop detailed rules and regulations. For the clinical test centre has not yet organized room quality assessment of clinical testing projects, should also establish room quality assessment system to ensure the quality of clinical testing.

\subsection{Clinical laboratory personnel should strengthen exchanges and improve their own quality}

Some of the clinical testing staff that quality control is not their own nature of work, little knowledge of quality control do not pay attention, leading to failure to implement quality control. In fact, clinical testing is a fine work. Test results, the difference between the least $\$$, the diagnosis may be absurd, ranging from delay to illness, while the disability and non-life $\%$, so the spirit of the work to concentrate, rigorous, orderly, meticulous, must not so-so. Medicine is to maintain personal safety and the promotion of human health science, each medical workers must be anxious patients, pain patients pain, as a qualified medical workers to treat any specimen should be extremely responsible, Not only to attach importance to the operation of a variety of instruments, should also attach importance to quality control of clinical testing, improve the quality control of the understanding, to provide accurate and reliable clinical test reports to lift the patient's pain, to contribute to the development of test medicine. 


\section{Conclusion}

Clinical examination is a comprehensive medical application science, involving a wide range, especially with the development of science and technology and testing methods have made a big progress and gradually improve the testing requirements. The automation degree of detection method is increasing, only strengthen the learning of clinical testing related knowledge can we standardize operational procedures, accurate interpretation of test data for clinical diagnosis and treatment to provide more reliable data support.

\section{References}

[1] Control and countermeasure of quality control in the laboratory of grass - root hospital. Journal of Traditional Chinese Medicine, 55(12), pp.68-70, 2011

[2] Li Aiping. Experience and Experience of Improving the Quality of Primary Clinical Medicine Examination, Chinese Practical Medical Journal, 8 (5), pp.8791, 2013

[3] Chen Junzheng. Strengthening the quality control of clinical laboratory and clinical departments. Modern Journal of Laboratory Medicine, 12(10), pp. 18- 20, 2012

[4] Shiquan Quan. Talking about the Problems and Solutions in the Quality Control of Medical Inspection. Modern Journal of Laboratory Medicine, 9(6), pp.58- 61,2011

[5] Zhang Heping. Quality management of laboratory in primary hospital. Chinese community physician, 8(4), pp.85-87, 2014 